\title{
Short Communication: Optimal Random Regression Models for Milk Production in Dairy Cattle
}

\author{
Y. X. Liu, ${ }^{*}$ J. Zhang, ${ }^{*}$ L. R. Schaeffer,† R. Q. Yang, ${ }^{* 1}$ and W. L. Zhang‡ \\ ${ }^{*}$ School of Agriculture and Biology, Shanghai Jiaotong University, Shanghai, China \\ †Centre for Genetic Improvement of Livestock, Department of Animal \& Poultry Science, University of Guelph, Guelph, \\ ON, Canada N1G 2W1 \\ $\ddagger$ Shanghai Supercomputer Center, Shanghai, China
}

\begin{abstract}
Legendre polynomials of orders 3 to 8 in random regression models (RRM) for first-lactation milk production in Canadian Holsteins were compared statistically to determine the best model. Twenty-six RRM were compared using LP of order 5 for the phenotypic ageseason groupings. Variance components of RRM were estimated using Bayesian estimation via Gibbs sampling. Several statistical criteria for model comparison were used including the total residual variance, the log likelihood function, Akaike's information criterion, the Bayesian information criterion, Bayes factors, an information-theoretic measure of model complexity, and the percentage relative reduction in complexity. The residual variance always picks the model with the most parameters. The log likelihood and information-theoretic measure picked the model with order 5 for additive genetic effects and order 7 for permanent environmental effects. The currently used model in Canada (order 5 for both additive and permanent environmental effects) was not the best for any single criterion, but was optimal when considering all criteria.
\end{abstract}

Key words: random regression model, optimization, statistical criteria

Random regression models (RRM) using Legendre polynomials $(\mathbf{L P})$ have been applied to production traits in dairy cattle, and to growth traits in beef cattle and swine. For dairy cattle the regressions are on number of DIM for both additive genetic (ADD) and permanent environmental (PE) effects. The order of the LP in the RRM is important in that estimates of genetic parameters can differ with the order (Misztal et al., 2000). The choice of submodel for ADD or PE is the focus in finding an optimal RRM. Jamrozik et al. (1997) found that an LP of order 5 for ADD and PE had a slight advantage in terms of prediction error variances for daily yield,

Received August 30, 2005.

Accepted January 26, 2006.

${ }^{1}$ Corresponding author: runqingyang@sjtu.edu.cn compared with LP of order 3. Commonly, RRM with orthogonal polynomials outperform models with lactation curve functions having the same number of parameters for ADD and PE effects (Jamrozik and Schaeffer, 2002). Furthermore, orthogonal polynomials have reduced correlations among the estimated coefficients (Schaeffer, 2004). Meyer (2000) and Pool et al. (2000) showed that the orders of the orthogonal polynomials do not need to be equal for ADD and PE effects. Guo and Schaeffer (2002) compared 18 submodels using several statistical criteria and concluded that orthogonal polynomials of order 4 or 5 were appropriate for RRM. In previous studies on optimization of RRM, the orders of LP fitting ADD and PE effects were usually limited to less than 5 and the statistical criterion for the choice of models was not uniform. Besides random regressions, functions are needed to account for the phenotypic relationship between test-day records and DIM (Schaeffer, 2004), and these are usually nested within age and season of calving. The objective of this study was to expand the search for an optimal RRM model to LP of order 3 to 8 .

After edits, data were 90,023 first-lactation test-day milk yields on 10,130 Canadian Holstein cows calving from 1988 to 1999. Data were restricted to DIM between 5 and $330 \mathrm{~d}$ inclusive, to test-day milk yields between 1.5 and $90 \mathrm{~kg}$ inclusive, to the number of records per cow greater than 7 , and to the number of records within a herd test date greater than 10 . The number of herdtest day subclasses was 7,657. Pedigrees were traced back 3 generations or to unknown parents.

The single-trait RRM (Schaeffer, 2004) for test-day milk yields was

$$
y_{i j k t}=H T D_{i}+g(t)_{j}+r\left(a, t, m_{1}\right)_{k}+r\left(p e, t, m_{2}\right)_{k}+e_{i j k t}
$$

where $y_{i j k t}$ is milk yield on the $k t h$ animal at DIM $t$ belonging to the ith herd-test date subclass, and the $j$ th age-season grouping (age at calving, 4 groups, by season of calving, 3 seasons); $H T D_{i}$ is the effect of the herd-test date on test-day records taken on the same date in a herd; $g(t)_{j}$ are LP of order 5 that account for 
the phenotypic trajectory of the average observations across all animals belonging to the $j t h$ age-season of calving grouping; $r\left(a, t, m_{1}\right)$ and $r\left(p e, t, m_{2}\right)$ are submodels (LP) for ADD and PE effects, respectively, of order $m$, the number of covariables related to time; and $e_{i j k t}$ is assumed to follow a normal distribution with mean null and variance $\sigma_{e(t)}^{2}$. Different residual variances were allowed for 30 time intervals within the interval from 5 to 330 DIM, defined as 5-20 DIM, 21-30 DIM, . ., 281290 DIM, 291-305 DIM, and 306-330 DIM. Residual effects were uncorrelated both within and between individuals.

The 2 random regression effects, $\mathrm{ADD}$ and $\mathrm{PE}$, were characterized using LP of different orders, which ranged from 3 to 8 . The models were designated by $\mathrm{LP}_{\mathrm{mn}}$, where $m$ is the order for the ADD effects and $n$ is the order for the PE effects. Thus, LP35 is a model with LP of order 3 for ADD and of order 5 for PE effects; a total of $26 \mathrm{RRM}$ were compared. The choice of optimal RRM was based on statistical criteria. The following statistical criteria were used:

Total residual variance $(\mathbf{T R V})=\sum_{t=5}^{330} \sigma_{e(t)}^{2} ;$

$\log$ likelihood function $[\log (\mathbf{L})]=-2 \log \left(M L_{k}\right)$;

Akaike's information criterion (AIC): $A I C_{k}=$ $-2 \log \left(M L_{k}\right)+2 p_{k}$ (Akaike, 1973);

Bayesian information criterion (BIC): $B I C_{k}=$ $-2 \log \left(M L_{k}\right)+p_{k} \log (n)$ (Schwarz, 1998);

Information-theoretic measure of model complexity (ICOMP): $\operatorname{ICOMP}_{k}=-2 \log \left(M L_{k}\right)+C_{1}(\Sigma)$

(Bozdogan, 2000); and

Percentage relative reduction of complexity (PRRC):

$$
\begin{gathered}
P R R C_{k}=\left[\left(C_{1}\left(\Sigma_{k}\right)-C_{1}\left(\Sigma_{k R}\right)\right) / C_{1}\left(\Sigma_{k}\right)\right] \\
\times 100 \%(\text { Bozdogan, 2000), }
\end{gathered}
$$

where $p_{k}$ is the number of free parameters in model $k$; $n$ is the number of observations that contribute to the likelihood; $\Sigma_{k}$ and $\Sigma_{k R}$ are covariance and correlation matrices of the parameters of model $k$, and $C_{1}(\Sigma)=$ rank $(\Sigma) \log [\operatorname{trace}(\Sigma) / \operatorname{rank}(\Sigma)]-\log (|\Sigma|)$.

Bayesian factor $(\mathbf{B F}): B F_{01}=p\left(y \mid M=M_{0}\right) / p\left(y \mid M=M_{1}\right)$ (Kass and Raftery, 1995), provides a contrast of model $M_{0}$ against model $M_{1}$, where $p\left(y \mid M=M_{k}\right)$ is an integrated (marginal) likelihood. According to Kass and Raftery (1995), a BF value greater than 150 [or $2 \log \left(B F_{01}\right)$ greater than 10$]$ indicates very strong evi- dence in favor of model $M_{0}$. Generally, small values are favorable for each statistic except for PRRC and BF [or $\log (\mathrm{BF})]$, where large values are favored. For BF, the model LP65 was used for $M_{1}$.

The covariance matrices of additive genetic, permanent environment random regression coefficients and residual variances of all competing $R R M$ were estimated using the GIBBS package of DMU (Madsen and Jensen, 2000).

The results under each criterion are expressed as differences from the model giving the smallest value for TRV, $\log (\mathrm{L}), \mathrm{AIC}, \mathrm{BIC}$, and ICOMP (Table 1). The results for $\log (\mathrm{BF})$ are based on comparing each model to LP65, and PRRC is a percentage value where the higher value is preferred. Total residual variance decreased within the orders for the ADD as the order of the PE effects increased. The difference among all competing models was nonsignificant according to likelihood ratio tests. Based on AIC and BIC, the model with the least number of estimated parameters (LP33) was the best. Model LP57 performed best on $\log (\mathrm{L})$ and ICOMP, whereas LP65 and LP66 did best on $\log (\mathrm{BF})$ and PRRC, respectively. There was not unanimous agreement on the best submodel among the 7 comparison methods used. In general, models were improved when the order of LP for PE was higher than for the order of ADD.

Because each statistical criterion indicated a different model as being optimal, the problem becomes one of deciding upon a statistical criterion that is best for comparison purposes. There are advantages and disadvantages of each criterion. Therefore, an index was created that added together $\mathrm{TRV}, \log (\mathrm{L})$, the absolute value of $\log (\mathrm{BF})$, AIC, BIC, ICOMP, and the negative of PRRC. The numbers are shown in the last column of Table 1 under INDEX. The index shows that model LP55 gave the smallest value, and therefore was the optimal model. Model LP55 is the model used in practice (in Canada) based on subjective assessments of researchers. Perhaps a better weighting of the different criterion would give a better index for comparison, but eventually a choice has to be made. Usually, only 1 criterion would be used to compare models rather than 7 because of the work involved in computing. Given that the model used in Canada is actually a multipletrait model with 3 lactations and 4 traits per lactation, using a model with orders greater than LP55 may not be computationally feasible.

\section{ACKNOWLEDGMENTS}

The authors thank J. Jamrozik and Z. Guo for their helpful suggestions from previous works. This research 
Table 1. Model selection criteria of the competing random regression models with LP5 for age-season of calving grouping and different orders of Legendre polynomials (LP) for additive and permanent environment effects, respectively

\begin{tabular}{|c|c|c|c|c|c|c|c|c|}
\hline \multirow[b]{2}{*}{ Model } & \multicolumn{8}{|c|}{ Selection criteria ${ }^{1}$} \\
\hline & TRV & $\log (\mathrm{L})$ & $\log (\mathrm{BF})$ & $\mathrm{AIC}$ & $\mathrm{BIC}$ & ICOMP & $\operatorname{PRRC}(\%)$ & INDEX \\
\hline LP33 & 120.5 & 12.7 & -94.2 & 0.0 & 0.00 & 24.4 & 61.28 & 190.5 \\
\hline LP34 & 84.8 & 19.5 & -198.7 & 14.8 & 26.6 & 38.3 & 61.21 & 321.5 \\
\hline LP35 & 61.1 & 14.1 & -141.5 & 19.4 & 46.0 & 27.6 & 59.23 & 250.5 \\
\hline LP36 & 43.5 & 6.7 & -108.4 & 24.0 & 68.3 & 12.9 & 59.13 & 204.7 \\
\hline LP37 & 21.4 & 6.4 & -52.9 & 37.6 & 102.6 & 12.4 & 54.24 & 179.1 \\
\hline LP43 & 92.9 & 10.3 & -244.5 & 5.6 & 17.4 & 19.9 & 58.45 & 332.2 \\
\hline LP44 & 83.5 & 9.5 & -135.8 & 12.8 & 36.4 & 18.6 & 67.61 & 229.0 \\
\hline LP45 & 59.4 & 7.5 & -92.7 & 20.8 & 59.2 & 14.7 & 65.61 & 188.7 \\
\hline LP46 & 41.9 & 5.7 & -66.5 & 31.0 & 87.1 & 11.2 & 64.23 & 179.2 \\
\hline LP47 & 19.1 & 4.3 & -128.2 & 43.6 & 120.4 & 8.6 & 60.06 & 264.1 \\
\hline LP53 & 73.7 & 16.1 & -152.1 & 21.4 & 48.0 & 31.6 & 55.73 & 287.2 \\
\hline LP54 & 65.9 & 10.8 & -209.5 & 24.1 & 62.5 & 21.2 & 50.77 & 343.2 \\
\hline LP55 & 51.6 & 5.0 & -19.3 & 28.3 & 81.4 & 9.8 & 62.35 & 133.1 \\
\hline LP56 & 39.3 & 5.2 & -46.7 & 40.4 & 111.3 & 10.3 & 68.82 & 184.4 \\
\hline LP57 & 17.2 & 0.0 & -1.5 & 49.2 & 140.8 & 0.0 & 64.44 & 144.3 \\
\hline LP63 & 59.8 & 13.1 & -167.5 & 30.4 & 74.7 & 25.7 & 59.51 & 311.7 \\
\hline LP64 & 52.3 & 5.2 & -197.1 & 30.5 & 86.6 & 10.2 & 50.89 & 331.0 \\
\hline LP65 & 42.9 & 15.4 & 0.0 & 50.6 & 121.5 & 30.7 & 70.63 & 190.5 \\
\hline LP66 & 35.7 & 13.1 & -58.2 & 60.4 & 149.0 & 26.5 & 73.69 & 269.2 \\
\hline LP67 & 14.9 & 2.8 & -1.3 & 63.4 & 172.7 & 4.5 & 70.28 & 189.3 \\
\hline LP73 & 43.8 & 6.0 & -125.7 & 37.3 & 102.2 & 11.6 & 58.58 & 268.0 \\
\hline LP74 & 35.4 & 9.5 & -119.9 & 48.7 & 125.5 & 18.8 & 64.27 & 293.5 \\
\hline LP75 & 27.9 & 6.8 & -55.2 & 56.1 & 147.7 & 13.7 & 69.15 & 238.2 \\
\hline LP76 & 20.8 & 1.4 & -41.5 & 62.7 & 172.0 & 3.1 & 72.73 & 228.8 \\
\hline LP77 & 11.3 & 8.8 & -5.7 & 84.0 & 214.0 & 17.9 & 72.39 & 269.3 \\
\hline LP87 & 0.0 & 9.8 & -80.1 & 101.1 & 254.7 & 20.1 & 71.39 & 394.4 \\
\hline
\end{tabular}

${ }^{1} \mathrm{TRV}=$ Total residual variance; $\log (\mathrm{L})=\log$ likelihood function; AIC = Akaike's information criterion; $\mathrm{BIC}=$ Bayesian information criterion; ICOMP = Information-theoretic measure of model complexity; $\mathrm{PRRC}=$ percentage relative reduction of complexity; TRV, $\log (L), \mathrm{AIC}, \mathrm{BIC}$, and ICOMP are relative to their minimum values, respectively.

${ }^{2} \mathrm{INDEX}=\mathrm{TRV}+\log (\mathrm{L})+\operatorname{abs}[\log (\mathrm{BF})]+\mathrm{AIC}+\mathrm{BIC}+\mathrm{ICOMP}-\mathrm{PRRC}$.

was supported by the Agriculture Cooperation Funds of Shanghai Jiaotong University.

\section{REFERENCES}

Akaike, H. 1973. Information theory and an extension of the maximum likelihood principle. Pages 267-281 in Proc. 2nd Int. Symp. Information Theory. B. N. Petrov and F. Csaki, ed. Akademia Kiado, Budapest, Hungary.

Bozdogan, H. 2000. Akaike's information criterion and recent developments in information complexity. J. Math. Psychol. 44:62-91.

Guo, Z., and L. R. Schaeffer. 2002. Random Regression Submodel Comparison. 7th World Congr. Genet. Appl. Livest. Prod. Montpellier, France. CD-ROM Commun. No. 20-08.

Jamrozik, J., G. J. Kistemaker, J. C. M. Dekkers, and L. R. Schaeffer. 1997. Comparison of possible covariates for use in a random regression model for analyses of test day yields. J. Dairy Sci. 80:2550-2556

Jamrozik, J., and L. R. Schaeffer. 2002. Bayesian comparison of random regression models for test-day yields in dairy cattle. 7th
World Congr. Genet. Appl. Livest. Prod. Montpellier, France. CDROM Commun. No. 01-03.

Kass, R. E., and A. E. Raftery. 1995. Bayes factors. J. Am. Stat. Assoc. 90:773-795.

Madsen, P., and J. Jensen. 2000. A user's guide to DMU. Danish Institute of Agricultural Science, Research Centre Foulum. Denmark.

Meyer, K. 2000. Random regressions to model phenotypic variation in monthly weights of Australian beef cows. Livest. Prod. Sci. 65:19-38.

Misztal, I., T. Strabel, J. Jamrozik, J. E. A. Mantysaari, and T. H. Meuwissen. 2000. Strategies for estimating the parameters needed for different test day models. J. Dairy Sci. 83:1125-1134.

Pool, M. H., L. L. G. Janss, and T. H. E. Meuwissen. 2000. Genetic parameters of Legendre polynomials for first-parity lactation curves. J. Dairy Sci. 83:2640-2649.

Schaeffer, L. R. 2004. Application of random regression models in animal breeding. Livest. Prod. Sci. 86:35-45.

Schwarz, G. 1998. Estimating the dimension of the model. Ann. Stat. 6:127-132 